\title{
Errata
}

\section{Erratum: Wanner et al., Cold-Induced Thermogenesis and Inflammation-Associated Cold-Seeking Behavior Are Represented by Different Dorsomedial Hypothalamic Sites: A Three-Dimensional Functional Topography Study in Conscious Rats}

In the article "Cold-Induced Thermogenesis and Inflammation-Associated Cold-Seeking Behavior are Represented by Different Dorsomedial Hypothalamic Sites: A Three-Dimensional Functional Topography Study in Conscious Rats” by Samuel P. Wanner, M. Camila Almeida, Yury P. Shimansky, Daniela L. Oliveira, Justin R. Eales, Cândido C. Coimbra, and Andrej A. Romanovsky, which appeared on pages 6956-6971 of the July 19, 2017 issue, the abbreviation VMHDM on page 6970 (paragraph 3, sentence 4) was attributed to a wrong neuroanatomical structure. The correct attribution is to the dorsomedial part of the ventromedial hypothalamic nucleus, and the sentence in question should read: "Remarkably, this behavioral sequence can be triggered by disinhibiting neuronal clusters in the ventral DM or in the dorsal part of the VMH (VMHDM) with bicuculline (Freitas et al., 2013; Dos Anjos-Garcia et al., 2016)." This correction does not affect the conclusions of the paper.

DOI:10.1523/JNEUROSCI.3541-17.2017

\section{Erratum: Machado et al., Conditional Deletion of Ric-8b in Olfactory Sensory Neurons Leads to Olfactory Impairment}

In the article "Conditional Deletion of Ric- $8 b$ in Olfactory Sensory Neurons Leads to Olfactory Impairment" by Cleiton F. Machado, Maira H. Nagai, Cassandra S. Lyra, Thiago M. Reis-Silva, André M. Machado, Isaias Glezer, Luciano F. Felicio, and Bettina Malnic, which appeared on pages 12202-12213 of the December 13, 2017 issue, the fifth author's name is incorrect. The corrected author list is as follows: Cleiton F. Machado, Maira H. Nagai, Cassandra S. Lyra, Thiago M. Reis-Silva, André Machado Xavier, Isaias Glezer, Luciano F. Felicio, and Bettina Malnic. The author line has been corrected on the online PDF version.

DOI:10.1523/JNEUROSCI.3582-17.2017 